\title{
Gastric adenocarcinoma concurrent with paravertebral plasmacytoma: A case report
}

\author{
FENGCAI DU $^{1,2}$, LIXIN JIANG ${ }^{3}$, FANGQING ZHU $^{1,2}$, ZHAO HUA GONG $^{1}$, \\ ${\text { JIAN } \mathrm{CHEN}^{1} \text { and LIANGMING ZHANG }}^{1}$
}

\begin{abstract}
${ }^{1}$ Oncology Department, Qingdao University Affiliated Hospital - Yantai Yuhuangding Hospital, Yantai, Shandong 264000;
${ }^{2}$ First Clinical Department of Graduate Academy, Dalian Midical University, Dalian, Liaoning 116000;

${ }^{3}$ Gastrointestinal Thyroid Surgery Department, Yantai Yuhuangding Hospital, Yantai, Shandong 264000, P.R. China
\end{abstract}

Received April 1,2015; Accepted April 22, 2016

DOI: $10.3892 / 01.2016 .4769$

\begin{abstract}
Here, we report the case of a 77-year-old male patient who was revealed to have an unsuspected case of gastric adenocarcinoma with paravertebral plasmacytoma following biopsy. Plasmacytoma may be classified into two main groups: Multiple myeloma and plasmacytoma without marrow involvement. It comprises isolated plasmacytoma of the bone and extramedullary plasmacytoma. Extramedullary plasmacytoma (EMP) accounts for $3 \%$ of all plasmacytomas; however, $\sim 80 \%$ are located in the upper respiratory tract and upper gastrointestinal tract. It occurs extremely rarely in paravertebral areas. Case reports of EMP and other types of malignant tumor occurring at the same time have not been identified in searches of the literature. In the present study, we describe the diagnosis and treatment process of a case of gastric adenocarcinoma concurrent with paravertebral plasmacytoma. It may be helpful for early clinical diagnosis and treatment of such cases.
\end{abstract}

\section{Introduction}

Extramedullary plasmacytoma (EMP) is an extremely rare type of plasmacytoma that is most similar to multiple myeloma. EMP accounts for $\sim 3 \%$ of all plasmacytomas and $\sim 1 \%$ of all general malignancies. It mainly occurs in the upper respiratory tract and upper gastrointestinal tract (80-90\%) (1). Cases of EMP generally present as masses with sections of nonspecific soft tissue density (2). The diagnosis of EMP

Correspondence to: Dr Zhao Hua Gong or Dr Jian Chen, Oncology Department, Qingdao University Affiliated Hospital - Yantai Yuhuangding Hospital, 20 Yuhuangding East Road, Yantai, Shandong 264000, P.R. China

E-mail: gongzhaohuayt@163.com

E-mail: chenjianyt@163.com

Abbreviations: EMP, extramedullary plasmacytoma

Key words: gastric adenocarcinoma, paravertebral plasmacytoma, concurrent requires rigorous investigation to rule out multiple myeloma and osteolytic lesions: Immunohistochemical analysis and biopsy/bone marrow puncture ( $<5 \%$ of plasmacytoid atypia) are performed to rule out multiple myeloma; serum and urinary protein electrophoresis are performed to detect $\mathrm{M}$ and Bence-Jones protein (no expression), respectively, to rule out osteolytic lesions. In addition, it is essential to ensure that the patient is not anemic (2-7). Treatment for EMP includes radiotherapy with high radiosensitivity (80-100\%) and surgery for localized lesions $(8,9)$; however, treated patients exhibit a recurrence or dissemination rate of $20-40 \%$ (8). In the present study, we report the case of a 77-year-old male patient who was revealed to have an unsuspected case of gastric adenocarcinoma with paravertebral EMP following biopsy. Following a search of the literature in PUBMED and the China National Knowledge Infrastructure (CNKI), no studies of EMP in the paravertebral area could be identified, indicating that our case may be unique. The process of diagnosis and treatment conforms to the ethics policy of our hospital.

\section{Case report}

Patient presentation and diagnosis. A 77-year-old male was admitted to Yantai Yuhuangding Hospital (Yantai, China) presenting with continuous pain in the epigastrium for months. He had a red blood cell count of $2.80 \times 10^{12} / 1$ and a hemoglobin level of $90 \mathrm{~g} / \mathrm{l}$. The patient had a long-term history of anemia. His serum calcium level was $1.87 \mathrm{mmol} / \mathrm{l}$. A urine analysis for Bence Jones proteins was negative. Gastroscopy examination revealed raised masses on the gastric antrum, an uneven surface, a hard, nodular feeling, and congestion over an area of $\sim 4 \times 3 \mathrm{~cm}$. Gastric ulcers and atrophic gastritis were visible (Fig. 1). Gastric biopsy revealed an epithelial coating, with visible effusion on the surface and diffuse infiltration of glands with atypical hyperplasia. Solid cell nests and single scattered cells were observed, as well as tumor cells infiltrating through the muscularis, which conformed with moderately differentiated adenocarcinoma of the gastric body (with a small number of cells having neuroendocrine differentiation; Fig. 2). A paravertebral mass was visible in abdominal computed tomography (CT), abdominal ultrasound and abdominal magnetic resonance imaging. Abdominal CT revealed a crumby mass 


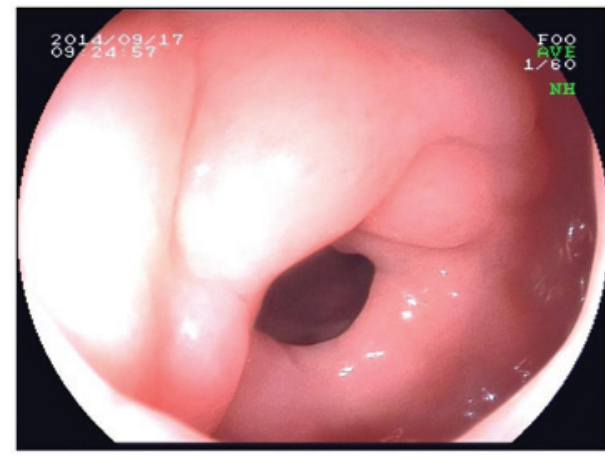

Figure 1. Gastroscopy revealed raised masses on the gastric antrum, an uneven surface, a hard, nodular sensation, and congestion over an area of $\sim 4 \times 3 \mathrm{~cm}$.

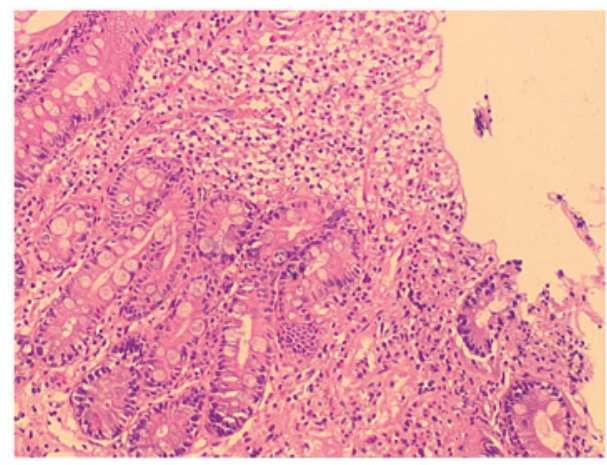

Figure 2. Gastric biopsy revealed an epithelial coating with visible effusion on the surface and diffuse infiltration of glands with atypical hyperplasia. Solid cell nests and single scattered cells were observed, as well as tumor cells infiltrating through the muscularis, conforming with a diagnosis of moderately differentiated adenocarcinoma of the gastric body. Hematoxylin and eosin staining; magnification, $\mathrm{x} 100$.

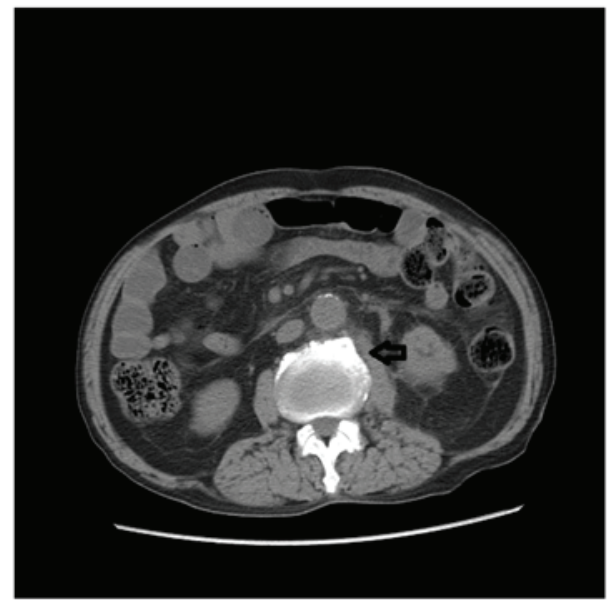

Figure 3. Abdominal computed tomography revealed a crumby mass with soft tissue density on the left of the 3-4 lumbar vertebra and abdominal aorta.

with soft tissue density on the left of the 3-4 lumbar vertebra and abdominal aorta (Fig. 3). Biopsy of the paravertebral mass revealed proliferation and infiltration of a large number of plasmocytes in adipose fiber connective tissue (Fig. 4). The plasma cells had extensive basophilic cytoplasms, and round eccentric nuclei with pleomorphism. Immunohistochemical

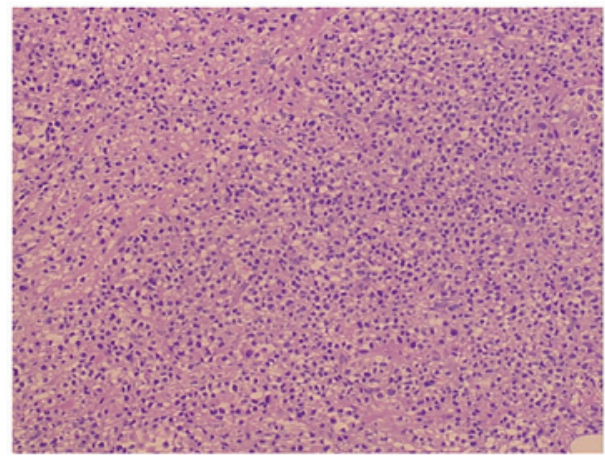

Figure 4. Biopsy of the paravertebral mass revealed proliferation and infiltration of a large number of plasmocytes in adipose fiber connective tissue. Hematoxylin and eosin staining; magnification, x100.

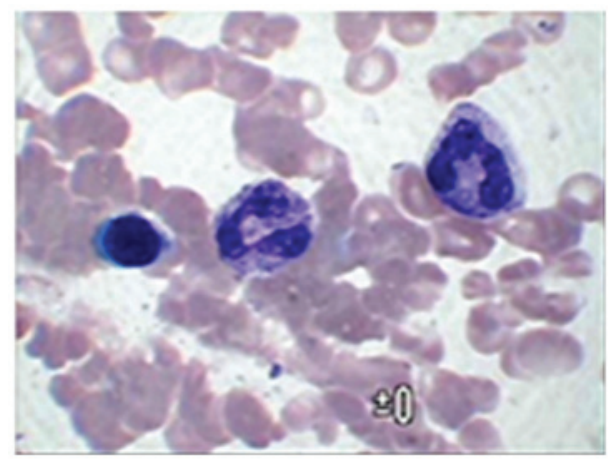

Figure 5. Bone marrow biopsy revealed no excess of plasma cells. Wright's staining; magnification, x100.

staining revealed that the plasma cells were negative for CD20, CD21, CD3, CD10, CD30, Bcl-6, Ig $\lambda$, CK and EMA. The plasma cells were positive for D138, mum1 and $\operatorname{Ig} \kappa$. The positive rate of $\mathrm{Ki}-67$ was $80 \%$. A bone marrow biopsy was performed, and no excess plasma cells were observed (Fig. 5). The histology and immunohistochemical staining results supported the diagnosis of EMP.

Treatment. The patient refused surgery. After signing the informed consent form, radiotherapy was administered to the patient to treat paravertebral plasmacytoma. Four cycles of oxaliplatin (200 mg, day 1; Cenexi, Fontenay-sous-Bois, France) plus tegafur, gimeracil and oteracil potassium capsules (40 mg, days 1-14; Taiho Pharmaceutical Co., Ltd., Tokyo, Japan) and zoledronate (Novartis Pharma Schweiz AG, Risch-Rotkreuz, Switzerland) were administered. The patient responded well to radiation and chemotherapy, and remains healthy with no evidence of tumor recurrence or secondary neoplasms one year after the initial diagnosis.

\section{Discussion}

The diagnosis and treatment process of this patient raises two key issues. Firstly, the early clinical symptoms are not typical; this may lead to misdiagnosis, although comprehensive diagnosis methods (combining imageology with pathology) are able to improve the diagnostic accuracy. Secondly, surgery is the most effective treatment if the conditions allow this. It is 
useful to combine local radiotherapy with chemotherapy when surgery cannot be performed. In addition, local radiotherapy may be of particular significance.

Plasmacytoma may be classified as multiple myeloma, solitary plasmacytoma, EMP or plasma cell leukemia. More than $95 \%$ of these tumors are multiple myelomas (10). The incidence of EMP is extremely low, accounting for just $3 \%$ of all plasmacytomas; $80 \%$ of these occur in the upper respiratory or digestive tract (1). Therefore, our case of EMP concurrent with another type of malignant tumor was rare. Makis et al (11) reported a case of gastric and mesenteric plasmacytoma occurring following surgery for colonic carcinoma. However, the two carcinoma types did not exist at the same time in this case. Early diagnosis is difficult due to its unclear and non-specific early clinical symptoms, and misdiagnosis and missed diagnosis occur frequently. Our patient suffered with irregular abdominal pain for several months, which could be diagnosed easily as gastric carcinoma through gastroscopy and pathology. However, the diagnosis of paravertebral plasmacytoma is difficult. Domestic diagnostic criteria of EMP is not yet available. However, the key points of clinical diagnosis may be summarized as follows: i) An extramedullary lump comprised of single monoclonal plasma cells; ii) a normal histological bone marrow test result to eliminate multiple myeloma; iii) a normal bone check including long bone imageological examination to exclude isolated plasmacytoma of the bone; iv) no anemia, hypercalcemia or renal insufficiency caused by plasma cell disease; and v) a deficient or low level of monoclonal immunoglobulin in the serum or urine (12). Scott et al (13) proposed that it was essential to check the monoclonal immunoglobulin level in serum or urine for EMP. Following auxiliary examinations, the paravertebral mass of our patients was observed to be largely in line with the above key points of diagnosis summarized above.

To date, there is no standardized treatment for EMP. Alexiou et al (14) observed that the relapse-free survival time following comprehensive treatment (radiotherapy plus surgery), radiation therapy alone, and surgery alone were 300, 144 and 156 months, respectively, by retrospectively clinical control study. The difference of statistical analysis $(\mathrm{P}<0.05)$ was statistically significant. Alexiou et al summarized their experience and noted that surgical resection was the first choice of treatment where feasible. Radiation and chemotherapy may be administered to patients with metastasis or surgical contraindication. Dimopoulos et al (15) observed that sensitivity to radiotherapy is much higher than that to chemotherapy, and that fewer side effects are involved in plasmacytoma of the head and neck. The prognosis of EMP is not bad. Studies have revealed that the ten-year disease-free survival rate of EMP is $~ 70 \%$ (16). The incidence of EMP progressing to multiple myeloma within 10 years was $11-30 \%$ (17). Therefore, preventing the possibility of EMP leading to multiple myeloma following treatment is one of the key factors in improving the prognosis. Avilés et al (17) suggested that the majority of patients treated with adequate radiation therapy alone are likely to develop multiple myeloma within the first three years after diagnosis and treatment. Radiation therapy alone may induce certain unknown side effects which should be paid attention.

In conclusion, there are few, if any, studies in the literature describing gastric adenocarcinoma concurrent with paravertebral plasmacytoma. Publishing our case may aid in the recognition of this rare disease, thus avoiding misdiagnosis and inadequate treatment.

\section{Acknowledgements}

The present study was supported by the National Natural Science Foundation of China (grant no. 81071758), Shandong Science and Technology Development Project (grant no. 2015GSF118142), the Natural Science Foundation of Shandong Province Joint Programme (grant no.ZR2015HL069) and Yantai Yuhuangding Hospital Initiative Foundation for Young Scientist (grant no. 201402).

\section{References}

1. Doki T, Takeuchi O, Kaiho T, Tsuchiya S, Matsuzaki O and Miyazaki M: Primary isolated extramedullary plasmacytoma of the colon. Int J Colorectal Dis 23: 719-720, 2008.

2. Ooi GC, Chim JC, Au WY and Khong PL: Radiologic manifestations of primary solitary extramedullary and multiple solitary plasmacytomas. AJR Am J Roentgenol 186: 821-827, 2006.

3. Luh SP, Lai YS, Tsai CH and Tsao TC: Extramedullary plasmacytoma (EMP): Report of a case manifested as a mediastinal mass and multiple pulmonary nodules and review of literature. World J Surg Oncol 5: 123, 2007.

4. Nakayama K, Okada D and Koizumi K: Excision of extramedullary plasmacytoma in a hilar lymph node. Japan J Lung Cancer 46: 723-726, 2006.

5. Bertolami A, Henriques AC and Penha FG: Plasmocitoma extramedular. Arq Med ABC 30: 58-60, 2005.

6. Galieni P, Cavo M, Pulsoni A, Avvisati G, Bigazzi C, Neri S, Caliceti U, Benni M, Ronconi S and Lauria F: Clinical outcome of extramedullary plasmacytoma. Haematologica 85: 47-51, 2000.

7. Lee SY, Kim JH, Shin JS, Shin C, In KH, Kang KH, Yoo SH: A case of extramedullary plasmacytoma arising from the posterior mediastinum. Korean J Intern Med 20: 173-176, 2005.

8. Ching AS, Khoo JB and Chong VF: CT and MR imaging of solitary extramedullary plasmacytoma of the nasal tract. AJNR Am J Neuroradiol 23: 1632-1636, 2002.

9. Ferrari S, Tecchio C, Turri G, Richelli S, Ghimenton C, Monaco S and Todeschini G: Unusual case of solitary intraparenchymal brain plasmacytoma. J Clin Oncol 30: e350-e352, 2012.

10. Lee HY, Kim JI and Kim KN: Solitary plasmacytoma of the rib. Korean J Thorac Cardiovasc Surg 45: 269-271, 2012.

11. Makis W, Ciarallo A, Hickeson M and Lisbona R: Gastric recurrence of a primary colon plasmacytoma: staging and evaluating response to therapy with $18 \mathrm{~F}-\mathrm{FDG}$ PET/CT. Br J Radiol 85: e4-e9, 2012.

12. Soutar R, Lucraft H, Jackson G, Reece A, Bird J, Low E and Samson D; Guidelines Working Group of the UK Myeloma Forum; British Committee for Standards in Haematology; British Society for Haematology: Guidelines on the diagnosis and management of solitary plasmacytoma of bone and solitary extramedullary plasmacytoma. Br J Haematol 124: 717-726, 2004.

13. Scott FE, Dupont PA and Webb J: Plasmacytoma of the stomach: diagnosis with the aid of immunoperoxidase technique. Cancer 41: 675-681, 1978.

14. Alexiou C, Kau RJ, Dietzfelbinger H, Kremer M, Spiess JC, Schratzenstaller B and Arnold W: Extramedullary plasmacytoma: tumor occurrence and therapeutic concepts. Cancer 85 : 2305-2314, 1999.

15. Dimopoulos MA and Hamilos G: Solitary bone plasmacytoma and extramedullary plasmacy toma. Curr Treat Options Oncol 3: 255-259, 2002

16. Dimopoulos MA, Kiamouris C and Moulopoulos LA: Solitary plasmacytoma of bone and extramedullary plasmacytoma. Hematol Oncol Clin North Am 13: 1249-1257, 1999.

17. Avilés A, Huerta-Guzmán J, Delgado S, Fernández A and Díaz-Maqueo JC: Improved outcome in solitary bone plasmacytoma with combined therapy. Haematol Oncol 14: 111-117, 1996. 